\title{
Efeito da categoria reprodutiva sobre a fertilidade de éguas inseminadas com sêmen asinino diluído e resfriado a $5^{\circ} \mathrm{C}$ por 12 horas de armazenamento
}

\author{
[Effect of mare status on fertility of inseminated mares with jackass semen diluted and \\ cooled at $5^{\circ} \mathrm{C}$ for 12 hours] \\ R. Rossi ${ }^{1}$, J.M. Silva Filho ${ }^{2}$, M.S. Palhares ${ }^{2}$, M.M. Silva ${ }^{3}$, R.A. Martins ${ }^{4}$, C.L.A. Silva ${ }^{1}$ \\ ${ }^{1}$ Aluna de pós-graduação - EV-UFMG - Belo Horizonte, MG \\ ${ }^{2}$ Escola de Veterinária - Universidade Federal de Minas Gerais - EV-UFMG - Belo Horizonte, MG \\ ${ }^{3}$ Médica veterinária autônoma - Belo Horizonte, MG \\ ${ }^{4}$ Médico veterinário residente - EV-UFMG - Belo Horizonte, MG
}

\begin{abstract}
RESUMO
Estudou-se o efeito da categoria reprodutiva sobre a fertilidade de éguas inseminadas com sêmen asinino diluído, resfriado e armazenado. Os ciclos foram acompanhados por palpação retal e rufiação, sendo as inseminações realizadas às terças, quintas e sábados, a partir da deteç̧ão de um folículo de 3,0 a 3,5cm de diâmetro, em um dos ovários, até a ovulação. O sêmen de cinco jumentos da raça Pêga foi diluído nos diluidores de leite em pó desnatado-glicose ou glicina-gema de ovo, resfriado a $5^{\circ} \mathrm{C}$ e armazenado por 12 horas, sendo a dose inseminante de $400 \times 10^{6}$ espermatozoides móveis (no momento da diluição final, pré-resfriamento) depositada no corpo do útero. O diagnóstico de gestação foi realizado por meio de palpação transretal, rufiação e ultrassonografia, realizada a cada 14 dias. Os resultados de 195 ciclos, referentes a 141 éguas, foram agrupados de acordo com a categoria reprodutiva a que pertenciam: potra, égua solteira, égua parida e no "cio do potro". As taxas de concepção, ao primeiro ciclo, foram de $60,00 \%, 48,28 \%, 75,00 \%$ e $47,17 \%$ e, após quatro ciclos, de $61,54 \%, 47,13 \%, 54,76 \%$ e $47,17 \%$, na mesma ordem para as categorias descritas anteriormente. A categoria reprodutiva não teve efeito $(\mathrm{P}>0,05)$ sobre a fertilidade das éguas inseminadas com sêmen asinino resfriado, sendo as potras, éguas solteiras, éguas paridas e no "cio do potro" igualmente eficientes para o uso na reprodução.
\end{abstract}

Palavras-chave: jumento, categoria reprodutiva, sêmen resfriado, fertilidade

\begin{abstract}
The effect of the mare status on fertility of mares inseminated with diluted, cooled and stored jackass semen, was studied. The cycles were controlled by transrectal palpation and teasing, and mares were inseminated every Tuesday, Thursday and Saturday, from the detection of a follicle with 3.0 to $3.5 \mathrm{~cm}$ diameter in one of the ovaries until ovulation. The semen of five Pêga jackasses was diluted in skim milkglucose or in egg yolk-glycine extender and cooled at $5^{\circ} \mathrm{C}$ for 12 hours, with the inseminate dose of $400 \mathrm{x}$ $10^{6}$ motile spermatozoa (at the moment of the final dilution, before cooling). The inseminations were carried out in the uterine body. Pregnancy was detected using transrectal palpation, teasing and ultrasound exams every 14 days. The results of 195 cycles of 141 mares were grouped according to the mare status: maiden, barren, lactation or in foal heat. Pregnancy rates for the first cycle were $60.00 \%$, $48.28 \%, 75.00 \%$ and $47.17 \%$, and after four cycles, the pregnancy rates/cycle were $61.54 \%, 47.13 \%$, $54.76 \%$ and $47.17 \%$, respectively for maiden, barren, lactation and in foal heat mares $(P>0,05)$. The mare status did not affect pregnancy rates of mares inseminated with diluted and cooled jackass semen and were efficient to use on reproduction.
\end{abstract}

Keywords: jackass, mare status, cooled semen, fertility

Recebido em 23 de setembro de 2013

Aceito em 21 de agosto de 2014

E-mail: raissarossi@hotmail.com 


\section{INTRODUÇÃO}

As fêmeas equinas podem ser distribuídas em diferentes categorias reprodutivas, de acordo com o seu estado fisiológico e metabólico. Cada categoria, seja potra, égua solteira, égua parida ou no "cio do potro", possui suas particularidades, vantagens e desvantagens quanto ao seu desempenho reprodutivo.

Von Lepel (1975) descreveu potras e éguas gestantes com um potencial de fertilidade superior $(70-100 \%)$ ao de éguas que não conceberam na estação anterior (50-70\%), ao das vazias por mais de duas estações reprodutivas consecutivas (25-50\%) e bem superior ao daquelas susceptíveis às infecções e com idade avançada ( $<25 \%)$.

Da mesma forma, outros autores também descreveram a categoria de potras como de alta fertilidade (Oliveira et al., 1988; Silva, 1988; Naden et al., 1990; Ferreira, 1993; Morris e Allen, 2002), embora seus ciclos sejam associados a alto percentual de atresias foliculares (Silva, 1988; Palhares, 1989). No início da vida reprodutiva das fêmeas equinas a idade deve ser considerada e analisada, já que Mitchell e Allen (1975) e Lucas et al. (1991) observaram alta taxa de perdas gestacionais em fêmeas muito jovens, destacando como causa a imaturidade reprodutiva dessas. Alguns trabalhos têm demonstrado a necessidade de um período pós-puberdade para o completo desenvolvimento reprodutivo (Wilsher e Allen, 2002), pois se observa, na espécie equina, aumento considerável do tamanho dos órgãos genitais no decorrer da puberdade.

As fêmeas da espécie equina são singulares no que diz respeito ao retorno da atividade ovariana no pós-parto. Entre os animais domésticos de grande porte, as éguas apresentam o menor intervalo do parto ao estro ("cio do potro"), evento que ocorre num período de cinco a 20 dias após o parto, acompanhado por falhas de ovulação inferiores a 10\%. A involução do endométrio uterino também é rápida e está completa aos 14 dias pós-parto, aproximadamente, o que viabiliza uma concepção precoce em relação às outras espécies domésticas. O exame ginecológico das éguas, nesse período, é de extrema importância, pois complicações após o parto e acúmulo de fluido intrauterino são fatores prejudiciais à fertilidade
(Ginther, 1992). Assim, o aproveitamento do "cio do potro é vantajoso, caso não haja complicações no puerpério, por reduzir o período de serviço e aproveitar o momento de alto estímulo ovariano, evitando o anestro que possa ocorrer nos ciclos subsequentes, devido a um desbalanço energético e metabólico (Reiner et al., 1988).

Na literatura, alta fertilidade também tem sido observada em éguas paridas ou no cio do potro (Von Lepel, 1975; Morris e Allen, 2002), sendo o nascimento de um potro sadio a comprovação do potencial de fertilidade da fêmea. No entanto, o período lactacional pode ser prejudicial à fertilidade, devido às altas taxas de reabsorção embrionária e de perda gestacional nessas éguas (Merkt, 1966; Merkt e Günzel, 1979; ChevalierClément, 1989; Fernandes et al., 1995).

No que se refere às éguas solteiras, excluindo-se aquelas que não gestaram por erro de manejo, a falta de cobertura ou a inseminação artificial são apontadas como responsáveis pelos piores índices de fertilidade no rebanho, pois, mesmo sendo inseminadas em diferentes ciclos, ao longo da estação de monta, continuaram vazias. Devido ao sistema reprodutivo debilitado, susceptível às infecções, a égua permanece improdutiva por mais anos consecutivos, havendo reduzida probabilidade de concepção (Morris e Allen, 2002).

Em virtude de suas particularidades e dos diferentes potenciais de fertilidade inerentes às categorias reprodutivas, o objetivo deste estudo foi avaliar seu efeito sobre a fertilidade de éguas inseminadas com sêmen asinino diluído e resfriado.

\section{MATERIAL E MÉTODOS}

O estudo foi realizado de outubro de 2007 a janeiro de 2008, em Lagoa Dourada, Minas Gerais, na região Sudeste do Brasil. A fazenda/unidade experimental está localizada a 1124 metros de altitude, $20^{\circ} 54^{\prime} 50^{\prime \prime}$ de latitude $\mathrm{S}$ e $44^{\circ} 4^{\prime}$ ' $40^{\prime \prime}$ de longitude $\mathrm{W}$.

Foram utilizadas 141 éguas mestiças, de 2,5 a 19 anos de idade, que resultaram em 195 ciclos estrais inseminados. As éguas foram agrupadas de acordo com a categoria reprodutiva a que pertenciam e analisadas quanto à fertilidade, sendo: potra (éguas jovens que nunca gestaram; 
$n=13$ ), égua solteira (vazia na estação anterior; $n=87$ ), parida (inseminada acima de 20 dias pósparto; $n=42$ ) ou no "cio do potro" (inseminada dentro de 20 dias pós-parto; $n=53$ ).

As éguas foram examinadas, por meio de palpação transretal, diariamente, complementadas pela rufiação, quando necessário, a partir da detecção de um folículo de 2,0 a 2,5cm de diâmetro, em um dos ovários. As inseminações foram realizadas a partir da detecção de um folículo com 3,0 a 3,5cm de diâmetro, em um dos ovários, até a ovulação, sendo o sêmen depositado no corpo do útero.

Para o diagnóstico de gestação, palpações transretais e rufiações de retorno foram realizadas, a partir do $15^{\circ}$ dia pós-ovulação, sendo complementadas pelo uso de ultrassonografia, realizada a cada 14 dias (SSD500, Aloka, probe de 7,5MHz). O diagnóstico por imagem iniciava-se num período mínimo de 11 dias pós-ovulação, sendo que cada égua positiva era submetida a, pelo menos, dois diagnósticos subsequentes. Das éguas que reabsorveram, apenas aquelas com período gestacional inferior aos 35 dias retornaram para o programa reprodutivo, de acordo com Penzhorn et al. (1986).

Foram utilizados cinco jumentos da raça Pêga, como doadores de sêmen, avaliados em um período anterior ao experimento para comprovação da qualidade do sêmen fresco e pós-resfriamento. O sêmen foi coletado por meio de vagina artificial modelo Hannover, as terças, quintas e sábados (três vezes/semana). Após a avaliação da motilidade e vigor espermáticos, procedeu-se à contagem das células espermáticas em câmara de Neubauer, para cálculo da concentração, com posterior diluição do sêmen em diluidor à base de leite em pó desnatadoglicose (Kenney et al., 1983) ou de glicina-gema de ovo (Foote, 2002). As doses inseminantes, de $20 \mathrm{~mL}$ e $400 \times 10^{6}$ espermatozoides móveis, foram resfriadas a $5^{\circ} \mathrm{C}$ por 12 horas de armazenamento, em contêiner especial (Palhares, 1997).

A fertilidade das éguas não foi influenciada $(\mathrm{P}>0,05)$ pelos diluidores de sêmen utilizados, pelo número de inseminações realizadas por ciclo, pelo intervalo IA-ovulação ou pela idade das éguas (Rossi, 2008). Assim, com esses fatores balanceados, foi possível analisar o efeito da categoria reprodutiva sobre a fertilidade das fêmeas equinas, agrupando-se os dados segundo a categoria à qual pertenciam: potra, égua solteira, égua parida ou égua no “cio do potro”, o que resultou em número diferente de ciclos para cada grupo.

A eficiência de prenhez foi calculada pela conversão de dados qualitativos em quantitativos, segundo proposta de Voss et al. (1975). Os dados foram submetidos à análise de variância, sendo as médias comparadas pelo teste de Student-Newman-Keuls (SNK). Os dados proporcionais (número de ciclos/concepção, taxa de concepção/ciclo, taxa de concepção total e ciclos/égua gestante) foram analisados por dispersão de frequência e comparados pelo teste de qui-quadrado para detecção de diferenças entre os grupos.

\section{RESULTADOS E DISCUSSÃO}

Observa-se, na Tab. 1, que as taxas de concepção ao primeiro ciclo, bem como as taxas de concepção/ciclo, foram similares $(\mathrm{P}>0,05)$ entre as categorias reprodutivas.

Como esperado, a idade inerente à categoria de potras foi inferior $(\mathrm{P}<0,05)$ às demais que, no entanto, foram similares $(\mathrm{P}>0,05)$ entre si (Tab. 2).

Os parâmetros reprodutivos (Tab. 2), tais como número de ciclos/concepção, concepção/ciclo, eficiência de prenhez, número de IA/ciclo e número de IA/ciclo positivo ou negativo, também não diferiram $(\mathrm{P}>0,05)$ entre as categorias reprodutivas.

O número de ciclos/égua e o número de ciclos/égua gestante foram inferiores $(\mathrm{P}<0,05)$ para as fêmeas da categoria “cio do potro" $(1,00$ e 1,00$)$, em relação às solteiras $(1,50$ e 1,39$)$ e as com potro ao pé $(1,67$ e 1,48), respectivamente. As potras foram similares $(\mathrm{P}>0,05)$ em ambos os parâmetros, às demais categorias. As éguas na categoria "cio do potro" foram inseminadas apenas no primeiro ciclo, já que mudaram de categoria logo no segundo ciclo pós-parto (Tab. 2), passando a constituir o grupo de éguas com potro ao pé. Tais mudanças explicam as diferenças observadas entre as categorias para as variáveis número de ciclos/égua e número de ciclos/égua gestante. 


\section{Rossi et al.}

Tabela 1. Efeito da categoria reprodutiva sobre a fertilidade, por ciclo estral, de éguas inseminadas com sêmen diluído e resfriado

\begin{tabular}{|c|c|c|c|c|c|c|c|c|c|c|}
\hline \multirow{3}{*}{ Ciclo } & \multicolumn{10}{|c|}{ Categoria reprodutiva } \\
\hline & \multicolumn{2}{|c|}{$\begin{array}{l}\text { Potra } \\
\text { (1) }\end{array}$} & \multicolumn{2}{|c|}{$\begin{array}{c}\text { Égua solteira } \\
(2)\end{array}$} & \multicolumn{2}{|c|}{$\begin{array}{l}\text { Potro ao pé } \\
\text { (3) }\end{array}$} & \multicolumn{2}{|c|}{$\begin{array}{c}\text { Cio do potro } \\
(4) \\
\end{array}$} & \multicolumn{2}{|c|}{ Total } \\
\hline & $\begin{array}{l}\text { № de } \\
\text { ciclos }\end{array}$ & $\begin{array}{c}\text { Taxa de } \\
\text { concepção } \\
\text { (\%) }\end{array}$ & $\begin{array}{l}\text { № de } \\
\text { ciclos }\end{array}$ & $\begin{array}{c}\text { Taxa de } \\
\text { concepção } \\
\text { (\%) }\end{array}$ & $\begin{array}{l}\text { № de } \\
\text { ciclos }\end{array}$ & $\begin{array}{c}\text { Taxa de } \\
\text { concepção } \\
\text { (\%) }\end{array}$ & $\begin{array}{l}\text { № de } \\
\text { ciclos }\end{array}$ & $\begin{array}{c}\text { Taxa de } \\
\text { concepção } \\
\text { (\%) }\end{array}$ & $\begin{array}{l}\text { № de } \\
\text { Ciclos }\end{array}$ & $\begin{array}{c}\text { Taxa de } \\
\text { Concepção } \\
(\%)\end{array}$ \\
\hline 1 & $10(6)^{\mathrm{a}}$ & 60,00 & $58(28)$ & 48,28 & 20(15) & 75,00 & $53(25)$ & 47,17 & 141(74) & 52,48 \\
\hline 2 & $2(2)$ & 100,00 & $22(10)$ & 45,45 & $16(6)$ & 37,50 & . & $\ldots$ & $40(18)$ & 45,00 \\
\hline 3 & $1(0)$ & 0,00 & $6(3)$ & 50,00 & $5(1)$ & 20,00 & $\ldots$ & $\ldots$ & $12(4)$ & 33,33 \\
\hline 4 & $0(0)$ & 0,00 & $1(0)$ & 0,00 & $1(1)$ & 100,00 & $\ldots$ & $\ldots$. & $2(1)$ & 50,00 \\
\hline Total & $13(8)$ & 61,54 & $87(41)$ & 47,13 & $42(23)$ & 54,76 & $53(25)$ & 47,17 & 195(97) & 49,74 \\
\hline
\end{tabular}

Números entre parênteses referem-se às fêmeas que conceberam

Tabela 2. Variáveis de éguas inseminadas com sêmen diluído e resfriado, agrupadas de acordo com a categoria reprodutiva

\begin{tabular}{lcccc}
\hline \multirow{2}{*}{ Variável } & \multicolumn{4}{c}{ Categoria reprodutiva } \\
\cline { 2 - 5 } № de éguas & Potra & Égua solteira & Potro ao pé & Cio do potro \\
№ de ciclos & 10 & 58 & 33 & 40 \\
Idade das éguas (anos) & 13 & 87 & 42 & 53 \\
№ de ciclos/égua & $3,23 \pm 0,78 \mathrm{~b}$ & $10,30 \pm 4,05 \mathrm{a}$ & $10,12 \pm 3,47 \mathrm{a}$ & $10,21 \pm 3,10 \mathrm{a}$ \\
№ de IA/ciclo & $1,30 \pm 0,67 \mathrm{ab}$ & $1,50 \pm 0,73 \mathrm{a}$ & $1,67 \pm 0,82 \mathrm{a}$ & $1,00 \pm 0,00 \mathrm{~b}$ \\
Volume de sêmen (mL) & $2,15 \pm 1,41$ & $1,94 \pm 1,00$ & $2,00 \pm 0,86$ & $2,02 \pm 0,75$ \\
Volume de diluidor (mL) & $3,22 \pm 1,45 \mathrm{ab}$ & $2,80 \pm 1,28 \mathrm{bc}$ & $2,32 \pm 1,29 \mathrm{c}$ & $3,50 \pm 2,13 \mathrm{a}$ \\
Tempo colheita/diluição (min) & $16,78 \pm 1,45 \mathrm{bc}$ & $17,22 \pm 1,29 \mathrm{ab}$ & $17,69 \pm 1,30 \mathrm{a}$ & $16,51 \pm 2,14 \mathrm{c}$ \\
Tempo colheita/resf. (min) & $20,25 \pm 4,60 \mathrm{a}$ & $18,64 \pm 2,73 \mathrm{~b}$ & $18,86 \pm 2,95 \mathrm{~b}$ & $19,22 \pm 3,39 \mathrm{ab}$ \\
Tempo colheita/abertura (h) & $13,10 \pm 0,64 \mathrm{ab}$ & $13,20 \pm 0,91 \mathrm{ab}$ & $13,35 \pm 0,74 \mathrm{a}$ & $12,99 \pm 0,56 \mathrm{~b}$ \\
Tempo colheita/IA (h) & $13,26 \pm 0,63 \mathrm{ab}$ & $13,35 \pm 0,92 \mathrm{a}$ & $13,49 \pm 0,75 \mathrm{a}$ & $13,16 \pm 0,58 \mathrm{~b}$ \\
№ de IA/ciclo positivo & $2,38 \pm 1,69$ & $1,78 \pm 0,85$ & $2,09 \pm 0,79$ & $2,04 \pm 0,79$ \\
№ de IA/ciclo negativo & $1,80 \pm 0,84$ & $2,09 \pm 1,11$ & $1,89 \pm 0,94$ & $2,00 \pm 0,72$ \\
№ de ciclos/concepção & 1,63 & 2,12 & 1,83 & 2,12 \\
Taxa de concepção/ciclo & 0,62 & 0,47 & 0,55 & 0,47 \\
№ de ciclos/égua gestante & $1,25 \pm 0,46 \mathrm{ab}$ & $1,39 \pm 0,63 \mathrm{a}$ & $1,48 \pm 0,79 \mathrm{a}$ & $1,00 \mathrm{~b}$ \\
Eficiência de Prenhez & $5,85 \pm 4,86$ & $4,34 \pm 4,71$ & $4,95 \pm 4,70$ & $4,72 \pm 5,04$ \\
\hline Médas sege
\end{tabular}

Médias seguidas por letras diferentes na mesma linha, diferem entre si $(\mathrm{P}<0,05)$

Quanto às variáveis volume de sêmen e volume de diluidor utilizados, observam-se diferenças $(\mathrm{P}<0,05)$ entre as categorias, em virtude de esses estarem relacionados à concentração espermática de cada ejaculado, com grande variação entre reprodutores, bem como entre ejaculados de um mesmo animal (Tab. 2).

Outras diferenças encontradas entre as categorias reprodutivas $(\mathrm{P}<0,05)$ envolveram os tempos da colheita/diluição, colheita/abertura e colheita/IA. Entretanto, a maior diferença foi de 1,61 minutos para tempo da colheita/diluição, e menor de 0,33 minutos para tempo da colheita/IA, consideradas desprezíveis na prática (Tab. 2).
As perdas gestacionais foram de 8,51\% (12/141), em todo o rebanho, com a seguinte distribuição por categoria reprodutiva: potra (1/12- 8,33\%), solteira (5/12- 41,67\%), "potro ao pé” (1/128,33\%) e "cio do potro" (5/12- 41,67\%). Apesar de o pequeno número de perdas gestacionais ter impedido a realização de análises estatísticas, observa-se que as éguas lactantes responderam por 50\% (6/12), e destas, 83,33\% (5/6) referemse às éguas no "cio do potro" e apenas 16,67\% (1/6) às fêmeas cobertas em cios subsequentes.

Tem sido observado que o incremento da fertilidade, em um rebanho equino pode ser verificado, ao longo de estações de monta consecutivas, pelo aumento da proporção de éguas com potro ao pé (lactantes) em relação à 
categoria de éguas solteiras (Silva, 1988). No presente trabalho, a distribuição de 141 fêmeas inseminadas, no que se refere às categorias reprodutivas, foi de $7,1 \%$ de potras; $41,13 \%$ de éguas solteiras e $51,77 \%$ de éguas lactantes representando, as últimas, um pouco mais da metade das fêmeas utilizadas. Entretanto, um rebanho com grande proporção de éguas em lactação, de acordo com Merkt (1966) e Merkt e Günzel (1979), seria motivo de preocupação em virtude do estado lactacional ser considerado prejudicial à fertilidade, devido à alta taxa de reabsorções embrionárias nesta categoria. Contudo, a taxa de perdas foi pequena no presente estudo, para todas as categorias estudadas, não demonstrando prejuízos no uso das éguas lactantes na reprodução.

Além disso, muitos autores têm verificado desempenho reprodutivo similar entre as diferentes categorias. Ferreira (1993), Fernandes et al. (1995) e Mattos et al. (1996), obtiveram taxas de gestação similares entre as categorias de potras, éguas solteiras e lactantes, o que está de acordo com os resultados do presente trabalho, no qual foi observada similaridade $(\mathrm{P}>0,05)$ entre as taxas de concepção ao primeiro ciclo, taxas de concepção/ciclo e eficiência de prenhez, considerando-se as diferentes categorias reprodutivas.

As potras apresentaram taxas de concepção similares às demais categorias, no presente experimento (Tab. 1 e 2 ). Tais resultados reforçam o conceito do alto potencial de fertilidade desta categoria, o que está de acordo com Nishikawa (1959), Von Lepel (1975), Oliveira et al. (1988) e Silva (1988), os quais não observaram restrição ao uso de potras de dois e três anos de idade na reprodução, obtendo taxas de fertilidade satisfatórias. Assim como no trabalho de Oliveira et al. (1988), as potras utilizadas no presente estudo apresentaram idade média acima de três anos de idade e bom escore corporal. Deve-se considerar que o número amostral de fêmeas (10) e de ciclos (13) para potras foi abaixo do ideal, para o teste de quiquadrado, o que pode causar sobreposição dos intervalos de confiança. No entanto, o presente experimento demonstrou a realidade de um rebanho para a produção de muares, com baixo número de potras. Ginther (1992) demonstrou que a categoria reprodutiva de potras representava a minoria (13\%) de diversos rebanhos na Inglaterra, França, Irlanda e Estados Unidos.

Em um trabalho realizado por Mattos et al. (1996), em que se utilizou a monta natural controlada ou a inseminação com sêmen fresco, foram obtidas taxas de gestação de 63,6\% e $71,4 \%$; de $52,9 \%$ e $92,8 \%$; e de $71,7 \%$ e $79,2 \%$, para potras, éguas falhadas e lactantes, respectivamente, e encontraram-se diferenças entre os métodos utilizados, apenas na categoria de éguas falhadas $(\mathrm{P}<0,05)$. Para éguas no "cio do potro”, a taxa de gestação obtida com a utilização da inseminação artificial foi superior à obtida com a monta natural (79,2\% vs 54,5\%, respectivamente). Outros autores também destacaram melhores resultados de fertilidade para éguas, quando foi utilizada a inseminação artificial associada a um melhor manejo reprodutivo veterinário, em relação àquelas que não a utilizaram (Kenney et al., 1975; Von Lepel, 1975; Pickett e Shiner, 1994; Morris e Allen, 2002). No presente estudo, foi utilizado serviço veterinário sistemático, associado às inseminações e a boas práticas de manejo e higiene, que podem ter sido responsáveis por amenizar possíveis problemas reprodutivos em éguas susceptíveis às infecções, e foram obtidas taxas de fertilidade similares entre as categorias reprodutivas.

\section{CONCLUSÃO}

A categoria reprodutiva não influenciou a fertilidade de éguas inseminadas com sêmen asinino resfriado.

\section{AGRADECIMENTOS}

À Fapemig pelo financiamento parcial deste trabalho; ao Sr. Renato Resende, pela cessão dos animais e instalações da Fazenda do Vau; à Hertape Calier e à Dra Marcela Roscoe, pelos medicamentos concedidos para o trabalho. 


\section{REFERÊNCIAS}

CHEVALIER-CLÉMENT, F. Pregnancy loss in the mare. Anim. Reprod. Sci., v.20, p.231-244, 1989.

FERNANDES, C.; CARNEIRO, U.; BORGES, C. et al. Eficiência reprodutiva de éguas PSI no Rio Grande do Sul. In: CONGRESSO BRASILEIRO DE REPRODUÇÃO ANIMAL, 11., 1995, Belo Horizonte. Anais... Belo Horizonte: Colégio Brasileiro de Reprodução Animal, 1995. p.389.

FERREIRA, M.F.L. Efeito de diluente e taxa de resfriamento sobre a motilidade espermática e fertilidade do sêmen de jumento (equus asinus). 1993. 94f. Dissertação (Mestrado em Medicina Veterinária) - Escola de Veterinária, Universidade Federal de Minas Gerais, Belo Horizonte, MG.

FOOTE, R.H. Whithin-Herd use of boar semen at $5^{\circ} \mathrm{C}$, with a note eletronic monitoring of oestrus. Reprod. Dom. Anim., v.37, p.61-63, 2002.

GINTHER, O.J. Reproductive biology of the mare: basic and applied aspects. Cross Plains, WI: Equiservices, 1992. 478p.

KENNEY, R.M.; BERGMAN, R.V.; COOPER, W.L. et al. Minimal contamination techniques for breeding. Technique and preliminary findings. Proc. Am. Assoc. Equine Pract., v.21, p.327-336, 1975.

KENNEY, R.M.; HURTGEN, J.P.; PIERSON, R. et al. Society for theriogenology manual for clinical fertility evaluation of the stallion. Hastings: Society for Theriogenology, 1983. 100p.

LUCAS, J.; RAESIDE, I.; BETTERIDGE, K.J. Non-invasive assessment of the incidences of pregnancy and pregnancy loss in feral horses of Sable Island. J. Reprod. Fertil., v.44, p.479-488, 1991.

MATTOS, R.C.; CAVALHEIRO, E.P.; MATTOS, R.C. et al. Monta natural e inseminação artificial com sêmen fresco diluído em éguas Árabe. Arq. Fac. Vet. UFRGS, v.24, p.57-64, 1996.

MERKT, $\mathrm{H}$. Foal heat and embryonic resorption. Zuchthygiene, v.1, p.102-108, 1966 apud Animal Breeding Abstracts, v.35, p.195. 1967.
MERKT, H.; GÜNZEL, A.R. A survey of early pregnancy losses in West German Thoroughbred mares. Equine Vet. J., v.11, p.256-258, 1979.

MITCHELL, D.; ALLEN, W.R. Observations on reproductive performance in the yearling mare. $J$. Reprod. Fertil., v.23, p.531-536, 1975.

MORRIS, L.H.A.; ALLEN, W.R. Reproductive efficiency of intensively managed thoroughbred mares in newmarket. Equine Vet. J., v.34, p.5160, 2002.

NADEN, J.; SQUIRES, E.L.; NETT, T.M. Effect of maternal treatment with altrenogest on age at puberty, hormone concentrations, pituitary response to exogenous $\mathrm{GnRH}$, oestrous cycle characteristics and fertility of fillies. J. Reprod. Fertil., v.88, p.185-195, 1990.

NISHIKAWA, Y. Development of the reproductive glands and sexual maturity in horses. In: STUDIES on reproduction in horses singularity and artificial control in reproductive phenomena. Tokio: Japan Racing Association, 1959. Cap.1, p.21-26.

OLIVEIRA, F.R.A.P.; TONHATI, H.; TOLEDO, L.R.A. et al. Comportamento reprodutivo de potras das raças Mangalarga e Bretão. Boletim da Ind. Anim., v.45, p.141-155, 1988.

PALHARES, M.S. Avaliação da atividade ovariana e eficiência reprodutiva de potras $e$ éguas da raça Mangalarga Marchador. 1989. 89f. Dissertação (Mestrado em Medicina Veterinária) - Escola de Veterinária, Universidade Federal de Minas Gerais, Belo Horizonte, MG.

PALHARES, M.S. Influência de um novo container para o transporte do sêmen equino diluído e resfriado: I - Características termodinâmicas e funcionais, II - Desempenho reprodutivo de éguas inseminadas. 1997. $245 \mathrm{f}$. Dissertação (Doutorado em Ciência Animal) Escola de Veterinária, Universidade Federal de Minas Gerais, Belo Horizonte, MG.

PENZHORN, B.L.; BERTSCHINGER, H.J.; COUBROUGH, R.I. Reconception of mares following termination of pregnancy with prostaglandin F2 $\alpha$ before and after day 35 of pregnancy. Equine Vet. J., v.18, p.215-217, 1986. 
PICKET, B.W.; SHINER, K.A. Recent developments in artificial insemination in horses. Livestock Prod. Sci., v.40, p.31-36, 1994.

REINER, U.R.; YUNES, C.M.; REINER, C. Cio Do Potro. In: CONGRESSO BRASILEIRO DE REPRODUÇÃO ANIMAL, 7., 1987, Belo Horizonte. Anais... Belo Horizonte: Colégio Brasileiro de Reprodução Animal, 1988. p.400407.

ROSSI, R. Comparação de dois diluidores na fertilidade de éguas inseminadas com sêmen asinino a fresco ou resfriado. 2008. 209f. Dissertação (Mestrado em Medicina Veterinária) - Escola de Veterinária, Universidade Federal de Minas Gerais, Belo Horizonte, MG.
SILVA, M.I.F. Viabilidade do uso da inseminação artificial em equinos à nível industrial. In: CONGRESSO BRASILEIRO DE REPRODUÇÃO ANIMAL, 7., Belo Horizonte, 1988. Anais... Belo Horizonte: Colégio Brasileiro de reprodução Animal, 1988. p.47-63.

VON LEPEL, J.FRHR. Maintenance of fertility in the horse including artificial insemination. Equine Vet. J., v.7, p.97-101, 1975.

VOSS, J.L.; PICKETT, B.W.; BACK, D.G. et al. Effect of rectal palpation on pregnancy rate of nonlactanting, normaly cycling mares. J. Anim. Sci., v.41, p.829-834, 1975.

WILSHER, S.; ALLEN, W.R. The influences of maternal size, age and parity on placental and fetal development in the horse. Theriogenology, v.58, p.833-835, 2002. 